\title{
Tangence
}

\section{Bataille et Dostoïevski via Thibaudet, Gide, Chestov. Jalons Bataille and Dostoevsky via Thibaudet, Gide, Chestov. Milestones}

\section{Jean-François Louette}

Numéro 86, hiver 2008

Dostoïevski et le roman russe dans l'entre-deux-guerres. Bataille, Beucler, Bove, Miomandre, Morand, Némirovsky, Ramuz

URI : https://id.erudit.org/iderudit/018625ar

DOI : https://doi.org/10.7202/018625ar

Aller au sommaire du numéro

Éditeur(s)

Tangence

ISSN

1189-4563 (imprimé)

1710-0305 (numérique)

Découvrir la revue

Citer cet article

Louette, J.-F. (2008). Bataille et Dostoïevski via Thibaudet, Gide, Chestov. Jalons. Tangence, (86), 89-103. https://doi.org/10.7202/018625ar
Résumé de l'article

Grand amateur de romans russes dans les années 1920, Bataille a lu avec attention deux des intercesseurs de Dostoïevski en France, Thibaudet et Gide. Le premier, qui dessine aussi des chemins possibles pour l'entreprise littéraire de Bataille, met l'accent sur la problématique du parricide - et la profanation des figures parentales sera constante dans les romans de Bataille. Le second insiste sur l'opacité des personnages, leurs excès, sur un certain penchant vers "l'informe ", et sur le récit intitulé L'esprit souterrain (ou Le sous-sol) : autant de points que retiendra Bataille. Mais comptera surtout pour lui la lecture que donne Chestov, dans Les révélations de la mort, en 1923, du Sous-sol, et l'analyse qu'il fait de l'homme souterrain : de cette analyse, « Dirty " sera à la fois une mise en récit et une mise en excès. 


\title{
Bataille et Dostoïevski via Thibaudet, Gide, Chestov. Jalons
}

\author{
Jean-François Louette, \\ Université de Paris IV-Sorbonne
}

\begin{abstract}
Grand amateur de romans russes dans les années 1920, Bataille a lu avec attention deux des intercesseurs de Dostoïevski en France, Thibaudet et Gide. Le premier, qui dessine aussi des chemins possibles pour l'entreprise littéraire de Bataille, met l'accent sur la problématique du parricide — et la profanation des figures parentales sera constante dans les romans de Bataille. Le second insiste sur l'opacité des personnages, leurs excès, sur un certain penchant vers «l'informe», et sur le récit intitulé L'esprit souterrain (ou Le sous-sol) : autant de points que retiendra Bataille. Mais comptera surtout pour lui la lecture que donne Chestov, dans Les révélations de la mort, en 1923, du Sous-sol, et l'analyse qu'il fait de l'homme souterrain: de cette analyse, «Dirty» sera à la fois une mise en récit et une mise en excès.
\end{abstract}

Le monde slave au rut obscur. Drieu La Rochelle, État civil, 1921

Si l'on consulte la liste des emprunts de Georges Bataille à la Bibliothèque nationale ${ }^{1}$, on ne peut manquer d'être frappé par la place qui, dans ses lectures, revient au roman russe, entre 1922 et 1925 , c'est-à-dire au moment même où se joue l'entrée en littérature du futur auteur d'Histoire de l'œil. De manière emblématique, le premier livre qu'il emprunte - ou dont l'emprunt ait été enregistré, le 24 juillet 1922 — est de Dostoïevski: L'éternel mari. Deux ans plus tard, Bataille lit Eugène Onéguine (août 1924); puis, de février à juillet 1925, il se fait prêter Les possédés et Les âmes mortes. Dostoïevski encore est au programme de cet été-là :

1. Liste établie par Jean-Pierre Le Bouler et Joëlle Bellec Martini, Euvres complètes, Paris, Gallimard, 1988, t. XII. Désormais, les références à cet ouvrage seront indiquées par le sigle $O C$, suivi de la page.

Tangence, $\mathrm{n}^{\circ} 86$, hiver 2008, p. 89-103. 
Crime et châtiment, ainsi que Le sous-sol, d'ailleurs réemprunté au mois d'octobre... mais peut-être déjà lu dès 1923, sous l'influence de Nietzsche, qui faisait le plus grand cas de ce récit ${ }^{2}$. En tout cas, à la fin de 1923, Bataille a rencontré le philosophe russe Léon Chestov, qui l'a poussé à approfondir sa connaissance du grand romancier.

Se propose ainsi la matière d'une étude comparée qui n'a guère été faite, même si tout le monde, évidemment, a relevé que la première page du Bleu du ciel (achevé en 1935, bien qu'il ne parût qu'en 1957) place le roman dans l'orbe de Dostoïevski: «En tous points, la scène qui précéda cette orgie répugnante [...] fut digne de Dostoïevski ${ }^{3}$...» Sans prétendre examiner l'ensemble de ce dossier, je me contenterai, d'une part, de former des hypothèses sur la manière dont Bataille a pu lire deux des grands intercesseurs de Dostoïevski en France, Albert Thibaudet et André Gide; d'autre part, j'examinerai ce que sa thématique en général, et un récit comme "Dirty» (qui date sans doute de 1925 ou 1926) en particulier, doivent à Dostoïevski tel que le lit Léon Chestov.

\section{Géographie littéraire}

Ouvrons les célèbres Réflexions sur le roman - recueil posthume d'Albert Thibaudet, le grand critique géographe de l'entre-deux-guerres, amateur fervent d'images paysagères pour expliciter sa pensée (il oppose, par exemple, les «virages» de Radiguet aux "méandres" de Giraudoux). Il s'agit, en relisant certaines des chroniques de Thibaudet parues dans la Nouvelle Revue française, d'esquisser les chemins qu'ouvre, à un jeune homme qui décide d'écrire, le paysage littéraire des années vingt. Que lui apprend, que lui suggère le champ littéraire? Quelle littérature à faire, pour reprendre un concept de Sartre, se propose-t-elle à lui?

Une telle question est sans doute bien trop ambitieuse. Pourtant, d'un seul article de Thibaudet, paru dans la Nouvelle Revue française en novembre 1921 et intitulé "Du roman anglais ", je puis extraire les trois propositions suivantes, dont on va tout de suite voir la pertinence pour Bataille:

2. C'est la date et l'influence que retient Bernd Mattheus, dans Georges Bataille. Eine Thanotographie, Munich, Matthes und Seitz Verlag, 1984, t. I, p. 59.

3. Georges Bataille, Romans et récits, éd. dirigée par Jean-François Louette, Paris, Gallimard, coll. «Bibliothèque de la Pléiade», 2004, p. 113. 
1. En 1921, «en France comme en Angleterre et comme ailleurs, faire de la littérature c'est faire du roman ${ }^{4} »$. Vingt ans plus tôt, c'était faire du théâtre. Mais les temps ont changé. Bataille, comme beaucoup d'autres, sera romancier. Même s'il tente parfois de faire entrer des poèmes dans ses romans (voir L'impossible), et toujours une poésie paradoxale.

2. Le roman «tend à devenir un genre de plus en plus international», ce qui n'était guère possible à la poésie: "Un poète, surtout un poète lyrique, est borné à son pays, il ne se traduit pas. Un romancier, s'il trouve un bon traducteur, ne perd que peu à la traduction. Et la traduction même n'est pas nécessaire pour lui créer un public international ${ }^{5}$.» Bataille sera sensible à la littérature étrangère: anglo-saxonne (Conrad, D. H. Lawrence, puis Hemingway), et surtout russe.

3. Parmi les romanciers qui sont des «natures», c'est-à-dire qui fonctionnent "comme des réalités incorporelles, génératrices d'hommes ${ }^{6}$ " figure, notamment, Dostoïevski. Dans sa chronique du $1^{\text {er }}$ avril 1924, intitulée "Le roman domestique», Thibaudet salue «la première traduction complète et véritable des Frères Karamazov», aux Éditions Bossard; il met, comme de juste, l'accent sur un thème qui ne devait pas manquer de frapper Georges Bataille, et qui se résume dans cette question autour de laquelle, selon le critique, tourne tout ce roman: "Qui ne désire pas la mort de son père ${ }^{7}$ ? Profaner les figures parentales, une mère ou un Père (de préférence de l'Église): ce geste sera, en 1928, au cœur d'Histoire de l'ceil. Ce n'est pas non plus par hasard que l'un des premiers récits de Bataille que nous ayons conservé, "Évariste ${ }^{8}$ ", qui est surtout le premier à être, au double sens du terme, achevé, a pour centre la mort d'une fillette (terne mais innocente): souvenir des Possédés? Car cette mort ne suscite chez Évariste, qui est le père de l'enfant, en rien les sentiments attendus: par son rire, indiscernable d'un sanglot, qui fait la chute du texte, il profane l'enfance, d'une façon non identique, mais comparable à celle de Stavroguine dans sa célèbre confession.

4. Albert Thibaudet, Réflexions sur le roman, Paris, Gallimard, 1938, p. 156.

5. Albert Thibaudet, Réflexions, ouvr. cité, p. 153.

6. Albert Thibaudet, Réflexions, ouvr. cité, p. 156.

7. Voir Les frères Karamazov, Paris, Gallimard, coll. «Bibliothèque de la Pléiade», 1952.

8. Ce récit inédit a été découvert par Marina Galletti et publié dans les Romans et récits, ouvr. cité, p. 934-943. 
4. Une dernière proposition est à tirer d'un autre article, lui aussi paru dans la Nouvelle Revue française, et qui date de mars 1922: "Le roman du plaisir». Thibaudet y soutient qu'il n'y a "presque pas de littérature voluptueuse», du moins qui ait de la valeur. À ce regrettable phénomène, on peut, lisant cette chronique, mais aussi celle du mois suivant, assigner trois raisons. D'une part, le plaisir physique "ne se révèle pas susceptible d'expression littéraire». Trop intense? Trop corporel? Thibaudet ne le dit pas. D'autre part, "tout plaisir exprimé littérairement devient plaisir de style, et sa lumière propre s'efface dans cette lumière ${ }^{9} »$. Autrement dit, il faudrait choisir: soit bien écrire, soit écrire la volupté. Le plaisir de plume ne tolère pas la plume vouée au plaisir, celle qui répond à l'appel de la sensualité. Si je veux rendre la volupté physique, je perds le style. Si je veux le style, je laisse se perdre la volupté charnelle. Passons de l'auteur au lecteur: si je ne lis que d'une main (Rousseau), ajouterais-je au propos de Thibaudet, je ne lis que d'un oil, et ce n'est plus le bon. - Enfin, «de ce qui est écrit sur le plaisir nous ne dirons jamais que c'est profond»: car jamais il ne touche à «l'homme devant l'énigme de la vie ${ }^{10} »$. On voit le défi que ces pages dessinent pour un Bataille: à lui d'éviter ce que Thibaudet baptise les "polissonneries lugubres", comme L'art de jouir de La Mettrie; à lui de tenter de produire une littérature érotique qui à la fois exprime le plaisir charnel, soit bien écrite, et se révèle profonde.

Ainsi, au total, si l'on imagine - ce qui n'a rien d'improbable, bien au contraire - Bataille lecteur de la Nouvelle Revue française en général et de Thibaudet en particulier, une espèce de destin littéraire possible s'indique pour lui : être un romancier, un romancier à la Dostoïevski, un romancier du plaisir profond. Or, quoi de plus profond que la mort? D'où la célèbre phrase sur laquelle, en 1957, s'ouvrira L'érotisme, mais que Bataille a pensée bien plus tôt: "L'érotisme est l'approbation de la vie jusque dans la mort»...

\section{Thibaudet et Gide}

Revenons à Dostoïevski. Thibaudet n'est certes pas le premier à le louer en France. Il y a eu, avant lui et entre autres interces-

9. Albert Thibaudet, Réflexions, ouvr. cité, p. 163.

10. Albert Thibaudet, Réflexions, ouvr. cité, p. 170. 
seurs, André Suarès, dont le Dostoïevski parut aux Cahiers de la quinzaine, le 10 décembre 1911. Et l'on ne saurait naturellement oublier André Gide.

Mais d'abord, que dit Thibaudet de Dostoïevski? Comme Gide, il s'inscrit en faux contre les réticences de l'introducteur du roman russe en France, le vicomte Eugène-Melchior de Vogüé - tout en lui reprenant la conception du roman dostoïevskien comme roman-somme ${ }^{11}$. Mais sans entrer dans ce débat, je me contente ici de retenir deux remarques. D'une part, Thibaudet repère le "romanesque psychologique» de l'imprévu chez le romancier russe, l'absence de caractères fixés ${ }^{12}$. Sur ce point, on pourrait dire que Bataille ne suivra qu'à demi le modèle de Dostoïevski. Certes, bien malin qui pourrait définir une psychologie de Simone, dans Histoire de l'ail, ou de Troppmann, dans Le bleu $d u$ ciel, personnages qui agissent par impulsions plutôt que par raisons, personnages d'extériorisation forcenée plutôt que d'intériorité songeuse. Mais la quête de l'excès, ou de la transgression, ne rend-elle pas au bout d'un moment les personnages de Bataille un peu plus prévisibles que ceux du romancier russe? En tout cas, il ne se rencontre pas chez eux de ces retours soudains vers le Bien qui adviennent ex abrupto chez les grands criminels de Dostoïevski.

D'autre part, Thibaudet note que Dostoïevski écrit des «romans-sommes qui donnent non une sensation d'ordonnance mais une sensation de long fleuve vivant ${ }^{13} »$. Bataille romancier n'aura jamais cette abondance, et il affectionne au rebours la «maigreur» (c'est son mot) du style. Il se rapproche plus de ce que Thibaudet voit comme la tradition française du roman, et qu'il définit ainsi, en 1920: «Le roman français a toujours tendance à imiter la tragédie française, à éliminer ou tout au moins à ramasser la durée, à contracter le personnage dans une figure plastique, dans un caractère fixe, et son action dans la peinture d'une crise ${ }^{14}$." Ainsi, chez Flaubert, un être (Emma) se détruit. C'est la tradition dominante, puisque depuis soixante ans, aux yeux de Thibaudet,

11. E.-M. de Vogüé, Le roman russe, Paris, Plon, Nourrit et Cie, 1886.

12. Albert Thibaudet, "La psychologie romanesque», Nouvelle Revue française, Paris, $1^{\text {er }}$ août 1924, Réflexions, ouvr. cité, p. 214-215.

13. Albert Thibaudet, "La composition dans le roman", Nouvelle Revue française, Paris, $1^{\mathrm{er}}$ novembre 1922, dans Réflexions, ouvr. cité, p. 186.

14. Albert Thibaudet, «Le centenaire de George Eliot», Nouvelle Revue française, Paris, $1^{\text {er }}$ février 1920, dans Réflexions, ouvr. cité, p. 95. 
tout le roman français est bâti sur Madame Bovary. Les romans français seraient des "romans de la fatalité »: ils suivent "une destinée qui retombe ${ }^{15}$ » (alors que le roman anglais, à la George Eliot, serait roman de la responsabilité et de la volonté). Ce modèle de la tragédie est très actif dans les romans de Bataille, en particulier dans Le bleu du ciel, où le tragique est à la fois personnel, historique et cosmique. Mais ce point a déjà été largement traité par la critique ${ }^{16}$ - donc je passe.

Enfin, sur le plan de la technique romanesque, on peut penser que Thibaudet, par souci d'opposer tradition russe du roman (le fleuve) et tradition française (marquée par le modèle tragique), minore la forte théâtralisation des romans de Dostoïevski. Milan Kundera y insistait tout récemment, dans Le rideau, en commentant le début de L'idiot: "Jusqu'alors, une telle concentration d'événements en un temps et un espace aussi serrés ne pouvait se voir qu'au théâtre »; Dostoïevski pousserait à l'extrême cette poétique de la scène qui œuvrait déjà chez Scott et chez Balzac ${ }^{17}$. Or l'on ne rencontre pas, chez Bataille romancier, de grandes scènes dialoguées. Tout se passe comme si s'était produit chez lui un déplacement de la dramatisation: du théâtral vers le mystique - au sens non classique qu'il donne à ce dernier terme. La dramatisation, selon L'expérience intérieure, n'est ni théâtre "extérieur" ni recherche du salut, mais volonté de cesser de vivre "isolés et tassés", effort pour atteindre à la "vie ardente", tension vers «l'extrême du possible»- le tout supposant un renoncement à l'ordre du discours, à la servilité des mots, et le recours à "des images bouleversantes", ou, d'après une note pour Le coupable, à «de violentes images ${ }^{18}$ ». Ce que Bataille va essayer de poser à l'horizon de ses romans, ce sont donc "ces états d'extase qui échappent aux données classiques de la religion» - mais il retrouve alors Dostoïevski par un autre biais, puisqu'en 1946, dans

15. Albert Thibaudet, "Le roman de la destinée», Nouvelle Revue française, Paris, $1^{\mathrm{er}}$ avril 1920, dans Réflexions, ouvr. cité, p. 107.

16. Voir Gilles Ernst, Georges Bataille. Analyse du récit de mort, Paris, Presses universitaires de France, 1993, p. 128-129; et notre notice pour Le bleu du ciel dans l'édition de la Pléiade, ouvr. cité, p. 1051-1052.

17. Milan Kundera, Le rideau, Paris, Gallimard, 2005, rééd. coll. «Folio», 2006, p. 31-32.

18. OC, 1973 , t. V, p. 22-26 et p. 139; voici la note pour Le coupable: «j'avais moi-même imaginé et tenté une pratique dans laquelle le principe premier est que l'on tende à se faire éclater la tête au moyen de violentes images (supplices, guerres)»(OC, t. V, p. 527). 
l'article de Critique dont je tire cette dernière citation, il compte le romancier russe au nombre de ceux qui ont décrit de tels états ${ }^{19}$.

Le Dostoïevski d'André Gide parut chez Plon en 1923. Il rassemble des articles et des causeries antérieures. Là encore, j'en retiens les remarques qui ont pu, qui ont dû compter pour Georges Bataille. J'en dénombre cinq.

1. "Ses principaux personnages restent toujours en formation, mal dégagés de l'ombre», indique Gide à la fin de son allocution au Vieux-Colombier, prononcée en 1921 pour le centenaire de la naissance du romancier russe ${ }^{20}$. De Dostoïevski, Gide - pour Les fauxmonnayeurs - et Bataille - pour tous ses romans — ont recueilli ce qu'il faudrait nommer, en plusieurs sens, une leçon de ténèbres.

2. Chez les personnages, les sentiments sont "poussés à bout, exagérés jusqu'à l'absurde ${ }^{21} »$. Céline retrouvera en 1937, dans Bagatelles pour un massacre, la même idée, exprimée plus plaisamment ${ }^{22}$ : "Souvent l'on s'est demandé après lecture des auteurs russes, je veux dire des auteurs de la grande période (pas les larbins soviétiques), par exemple Dostoïewsky [sic], Tchekov [sic], même Poutchkine $[s i c]$, d'où ils provenaient ces hommes avec ces transes, comment ils tenaient à longueur d'œuvre le ton de cette rumination funèbre, délirante?» (La réponse de Céline est que ce type de personnages hante les rues, en Russie: «Raskolnikoff? Mais pour les Russes c'est du Bouboule!... » On appréciera mieux l'humour de Céline si l'on se souvient que Bouboule est le nom d'une dame, personnage récurrent de la romancière Trilby, qui écrivait de curieux romans du cœur engagés, une espèce de littérature à l'eau de rose politisée: ainsi Bouboule et le Front populaire, paru en 1937 chez Flammarion). - La transe, l'exagération, la rumination funèbre, le délire, voilà l'état perpétuel du protagoniste du Bleu du ciel, au nom transparent: Troppmann, homme du trop.

3. Gide fait une observation plus singulière lorsqu'il invite à constater que «presque tous les personnages de Dostoïevski sont polygames", par quoi il faut entendre: "capables simultanément de plusieurs amours», et étrangers à la jalousie ${ }^{23}$. Ainsi Tropp-

19. OC, t. XII, p. 183.

20. André Gide, Dostoüevski, Paris, Gallimard, coll. «Les essais», 1981, p. 63.

21. André Gide, Dostoïevski, ouvr. cité, p. 119.

22. Louis-Ferdinand Céline, Bagatelles pour un massacre, Paris, Denoël, 1937, p. 119-120.

23. André Gide, Dostoïevski, ouvr. cité, p. 127. 
mann est-il pris entre Dirty, Xénie, et accessoirement les filles de petite vertu qu'il fréquente. La Simone d'Histoire de l'oil ne semblait pas non plus vouée à la monogamie.

4. Plus original encore: dès 1908, à propos de la correspondance de l'écrivain russe, Gide en relève la "gaucherie si caractéristique»: «le texte de ces lettres est confus, maladroit, incorrect ${ }^{24} »$. Avec Dostoïevski se joue la possibilité d'un adieu à l'éloquence et à l'apprêt. Gide emploie alors un mot qui ne peut manquer de sonner haut et fort à l'oreille de tout lecteur de Bataille: il parle de "l'énorme informité " de chacune de ses lettres ${ }^{25}$. En cette informité qui, maîtrisée, deviendra la puissante complexité des grands romans de Dostoïevski, comment ne pas reconnaître une première figure de cet informe auquel Bataille consacrera un article célèbre du «Dictionnaire» de Documents, en 1929 ? D'autant que Gide emploie ce terme exact plus loin dans son livre de 1923: le désordre de Dostoïevski, il faut éviter de le condamner hâtivement, au motif que le génie français (ordre, clarté, logique, etc.) a "horreur de l'informe ${ }^{26} »$. La différence entre Gide et Bataille éclate cependant: le premier s'empresse de ne pas aller trop loin — «d'abord Dostö̈evski n'est pas informe; loin de là: tout simplement ses codes de beauté sont différents de nos codes méditerranéens"; tandis que le second, dans le cadre d'une esthétique qui récuse l'opposition entre laideur et beauté, prendra le risque de fonder son œuvre romanesque sur - le paradoxe est là essentiel — un certain apprêt de l'informe et de la gaucherie ${ }^{27}$. "Un peu blessée j'écris main gauche»: cette formule d'un personnage de L'impossible est décisive, en ce qu'elle articule la souffrance et la maladresse.

5. Le sommet de l'œuvre de Dostoïevski, sa "clé de voûte», pour Gide, c'est L'esprit souterrain ${ }^{28}$ (titre sous lequel était alors connu Le sous-sol). On a vu que Bataille donnait ce récit pour un intertexte capital du Bleu du ciel; et ce dès la version manuscrite de

24. André Gide, Dostö̈evski, ouvr. cité, p. 19.

25. André Gide, Dostö̈evski, ouvr. cité, p. 20.

26. André Gide, Dostö̈evski, ouvr. cité, p. 184.

27. Voir Francis Marmande, L'indifférence des ruines, Paris, Parenthèses, 1985, p. 77-78; et, pour une mise au point sur la dialectique de la gaucherie et de l'élégance, dans l'édition de la Pléiade, notre introduction (p. LXXVILXXVII), ainsi que la notice du Bleu du ciel, ouvr. cité, p. 1069-1070, et la description (p. 1103-1108) du travail stylistique par lequel Bataille passe de la version manuscrite de 1935 au texte publié en 1957.

28. André Gide, Dostoïevski, ouvr. cité, p. 133. 
1935. C'est encore d'un extrait de ce récit — l'épisode où l'homme du sous-sol rencontre Lise, une prostituée, dans une maison close - que Bataille et sa cousine germaine Marie-Louise prépareront une adaptation dialoguée, diffusée par la Radio française le 19 juin 1946, toujours sous le titre de L'esprit souterrain ${ }^{29}$. Mais au fond, c'est toute l'œuvre narrative de Bataille qui, se fondant sur des anti-héros - il faudrait préciser en quel sens ${ }^{30}$-, est traversée, comme celle du romancier russe, par la fascination de la méchanceté (Dostoïevski aurait certes été à sa place dans La littérature et le mal), du déclassement et de l'infamie: "Kierkegaard est l'extrême du chrétien. Dostoïevski (dans Le sous-sol) est l'extrême de la honte ${ }^{31}$." Bataille en 1943 résumera ainsi son entreprise: "Je pense, j'écris, pour ne connaître aucun moyen d'être mieux qu'une loque $^{32} »-$ et il faut donner à la conjonction pour son sens plein de but. En 1946, il louera Arthur Adamov d'avoir, dans L'aveu, retrouvé le ton exact du Sous-sol: l'abaissement, "une horreur colorée, que seul atténuerait l'élément grotesque, on ne sait quoi de grandiloquent qui serre le cœur ${ }^{33} »$. Quant à lui, à l'impulsion reçue de Dostoïevski, il imprime cette inflexion personnelle, et décisive: l'intérêt passionné pour "le bas matérialisme", sous toutes ses formes ${ }^{34}$. En ce sens, le titre du récit de Dostoïevski vaut pour Bataille comme un programme anti-idéaliste à remplir : l'esprit, il faut le faire descendre sur terre, voire sous terre. C'est ce qui apparaît au mieux dans un récit comme «Dirty».

\section{«Dirty», Chestov}

Le début du roman Le bleu du ciel reprend un récit de Bataille connu sous le nom de «Dirty». Son histoire est singulière: il paraît à l'extrême fin de 1945, mais en portant comme date, sous la dernière ligne, 1928 ; et selon Michel Leiris, il aurait formé le

29. Elle a été publiée par les soins de Cécile Moscovitz dans L'infini, Paris, $n^{\circ} 75$, été 2001, p. 53-79, avec une riche introduction intitulée «Bataille et l'homme du sous-sol», p. 46-52.

30. Dans Georges Bataille. Eine Thanotographie (ouvr. cité, p. 60), Bernd Mattheus rapproche du Sous-sol et de son anti-héros le roman que Bataille projetait d'écrire en 1923 et qui se serait intitulé «Le joyeux cynique».

31. Georges Bataille, L'expérience intérieure, OC, t. V, p. 56.

32. Georges Bataille, L'expérience intérieure, $O C$, t. V, p. 81.

33. Georges Bataille, Critique, Paris, nº 7, décembre 1946, dans OC, t. XI, p. 162.

34. Voir Georges Bataille, «Le bas matérialisme et la Gnose», Documents, Paris, 1930, repris dans OC, t. I. - Ce point a été bien vu par Cécile Moscovitz. 
premier chapitre d'un roman écrit en 1925 ou 1926, W. C., que Bataille par la suite détruisit, mais dont il aurait, donc, conservé de quoi faire l'amorce du Bleu du ciel $^{35}$. Ce chapitre a le prestige des commencements: on doit placer avant lui «La châtelaine Gentiane ", "Ralph Webb» et "Évariste», mais il ouvrait le premier roman que Bataille ait, un temps, voulu fermement publier. 1925 : c'est en juin de cette année, puis en octobre, que Bataille, je le répète, emprunte à la Bibliothèque nationale Le sous-sol. Or, voici la première phrase de «Dirty»: «Dirty était saoule à côté de moi; dans les sous-sols d'un bouge ignoble.»

Le récit se compose de deux scènes. Dans la première, une femme, Dirty, ivre et belle, montre son sexe dans un bouge, à Londres. La seconde scène se déroule dans une chambre de palace, le Savoy: Dirty évoque devant son ami Léon le souvenir d'une chute grotesque que fit sa mère dans cet hôtel. Ayant appelé une femme de chambre et un liftier, elle leur jette un énorme pourboire, puis urine et défèque devant eux. Une fois lavée, elle prend à partie les domestiques, criant sa peur et son écœurement. Enfin, elle vomit par la fenêtre, sous le regard de Léon, vide, angoissé.

On saisit à la première lecture ce qui donne au récit son côté Dostoïevski : l'atmosphère de frénésie, l'auto-humiliation en public, la prodigalité irraisonnée (on songe à Anastasie Filippovna jetant au feu cent mille roubles dans L'idiot), le couple de la prostituée et du criminel (sur le modèle de Sonia et Raskolnikov dans Crime et châtiment), même si Léon ne porte pas encore le nom de ce meurtrier célèbre, Troppmann, qui ne deviendra le sien que dans Le bleu du ciel.

En marquant cet intérêt pour Dostoïevski, Bataille passe par dessus - ce sont ses propres mots — "l'incompréhension purement brutale du Manifeste du surréalisme jugeant l'œuvre de Dostoïevski ${ }^{36}$ ». Se séparant de Breton, Bataille se place du côté de Chestov, qu'il a fréquenté de 1923 à 1925. C'est en effet aussi à travers les textes que lui consacre Léon Chestov que Bataille se familiarise avec l'œuvre de Dostoïevski, et le prénom Léon attribué au protagoniste de "Dirty» signale peut-être, en un clin d'œil, cette influence de Chestov.

35. Voir Michel Leiris, «De Bataille l'Impossible à l'impossible Documents", Brisées, Paris, Mercure de France, 1966, p. 258-259.

36. Georges Bataille, «Le temps de la révolte», OC, t. XII, 1951, p. 153. 
Je voudrais m'attarder sur une très belle étude, intitulée «La lutte contre les évidences (Dostoïevsky)», qui forme la première partie des Révélations de la mort, livre publié en 1923 par Chestov et emprunté par Bataille à la Bibliothèque nationale en août 1924 - année où il collabore à la traduction d'un autre ouvrage de Chestov, L'idée de bien chez Tolstoï et Nietzsche ${ }^{37}$. L'analyse de Chestov porte avant tout sur Le sous-sol, auquel la traduction de Boris de Schloezer donnait pour titre La voix souterraine. On ne peut qu'être frappé par l'influence de ces pages sur «Dirty» (et plus généralement sur l'ensemble de l'œuvre de Bataille). Le texte de Bataille constitue en un sens une mise en récit de l'analyse de Chestov - mais aussi une mise en excès, conformément à ce que l'écrivain notera plus tard: "j'eus vite l'impression de différer de lui en raison d'une violence fondamentale qui me portait ${ }^{38}$ ", violence d'ordre sexuel, notamment.

Chestov commence par établir une distinction entre deux visions. D'une part, la "vue naturelle», à laquelle se lie notre raison, et qui passe comme de juste par des "yeux naturels», ceux de tout le monde. D'autre part, la «seconde vision» octroyée à certains hommes par l'Ange de la Mort: ces "yeux surnaturels" donnent accès à de «nouvelles visions» qui paraissent «illégales, ridicules, fantastiques ", à la limite de la folie; elles se situent dans un espace où la vérité est délivrée de la certitude, et s'impose contre les évidences. Une histoire d'œil nouveau, donc. Aux cyclopes d'Homère il faudrait opposer... les di-opes ou di-opses de Chestov? En tout cas, cette "seconde paire d'yeux", cette «seconde vue» fut octroyée à Dostoïevski, et le mena à un certain nombre de découvertes, que j'énumère:

- D'abord, celle de la réversibilité entre vie et mort: "personne n'est certain que la vie ne soit pas la mort». - Une telle

37. On s'étonne que les Révélations de la mort, et cette étude en particulier, ne soient jamais citées dans un article de Michel Surya intitulé "L'arbitraire, après tout», sous-titré «De la "philosophie" de Léon Chestov à la "philosophie" de Georges Bataille», et qui, dans ses dernières pages, se penche sur la relation de Bataille à Dostoïevski; voir dans Denis Hollier (sous la dir. de), Georges Bataille après tout, Paris, Belin, 1995, p. 213-231. On lira en revanche, sur la triade Dostoïevski - Chestov — Bataille, les pages très bien informées de Philippe Sabot, Pratiques d'écriture, pratiques de pensée. Figures du sujet chez Breton, Éluard, Bataille et Leiris, Villeneuve d'Ascq, Presses universitaires du Septentrion, coll. "Problématiques philosophiques», 2001, p. 115-123; il montre notamment comment la littérature est, chez Bataille, le souterrain de la philosophie - l'espace où cette dernière s'effondre.

38. Note autobiographique (1958?), OC, t. VIII, 1976, p. 563. 
proposition paradoxale - je le répète, il faut avoir été visité par "l'Ange de la Mort» pour la comprendre - ne serait-elle pas à la source de l'extraordinaire valorisation bataillienne de la mort?

- Dostoïevski aurait compris aussi qu'il n'y a pas de ciel libérateur: "il n'y a de ciel nulle part; il n'y a qu'un "horizon” bas et borné. Les idéals qui élèvent l'âme n'existent pas, il n'y a que des chaînes, invisibles, il est vrai, mais qui maintiennent l'homme encore plus solidement que les fers ». - Bataille ne cessera de tempêter contre tous les mensonges de l'idéalisme et - dès sa polémique contre André Breton - de l'icarisme.

— C'est cette «"vision" nouvelle» qui forme le thème de $L a$ voix souterraine, récit où "l'homme souterrain» est oppressé par "un sentiment torturant du néant», à savoir, "la sensation très nette que cet état d'équilibre, de parfait achèvement, de satisfaction complète considéré par la conscience commune [...] comme l'idéal de la pensée humaine [...] est absolument insupportable». - De même, Bataille se débat continûment contre l'atroce geôle où l'enferment la raison (cartésienne) et la normalité (bourgeoise).

- Dès lors que toute l'expérience humaine « et ses limites, telles qu'elles apparaissaient à Kant, et à Comte, ne sont plus aux yeux de Dostoïevski que l'enceinte d'une prison bâtie pour nous par un inconnu", il ne se sent "soutenu par aucune autorité, par aucune tradition». - L'absence d'autorité: c'est un des grands thèmes de l'œuvre de Bataille, qui se manifeste par exemple dans sa contestation, par des pseudonymes (Lord Auch, Pierre Angélique...), de son propre nom d'auteur, ou dans son opposition à l'emphase et à la posture (de chef, de mage, de prophète, etc.) d'un André Breton.

- Dostoïevski est attiré, note encore Chestov, par «le "peutêtre", l'inattendu, le subit, les ténèbres, le caprice, tout cela justement qui, au point de vue de la science et du bon sens, n'existe pas ou ne doit pas exister». L'homme souterrain «se dresse», et d'une voix affreuse, "d'une voix qui n'est pas la sienne (la voix de l'homme souterrain, aussi bien que sa vue, ne lui appartiennent pas en propre)», il défend l'impossible, à la fois contre Kant ( $\mathrm{La}$ religion dans les limites de la simple raison) et contre le catholicisme (Deus impossibilia non jubet) : "Dieu exige l'impossible. Dieu n'exige que l'impossible [...] La métaphysique est impossible! Donc je ne penserai plus qu'à elle, je ne parlerai plus que d'elle.» Une «impulsion intérieure, plus puissante que les intérêts» refuse de considérer l'impossible comme une "muraille de pierre» 
(formée par le savoir, la science, les lois naturelles). — De l'impossible, Bataille fera une notion centrale (même si par définition elle est peu saisissable) de sa pensée ${ }^{39}$, et le titre d'un de ses romans les plus ambitieux (même si ce n'est pas le plus réussi).

— L'homme souterrain ira jusqu'à consentir à «l'inertie», il se laissera écraser, humilier, pour échapper à la contrée où règnent les lois, celle où vivent les gens normaux, modérés, aristotéliciens, qui suscitent «dans la région souterraine un rire homérique». - Le rire, expérience fondamentale et "question clé " (L'expérience intérieure) chez Bataille, qui reproche à Bergson, dès 1920, de n'y avoir rien compris ( «la théorie me sembla courte», toujours selon L'expérience intérieure)... Rire qu'on devrait aborder de bien des façons, mais dont il est essentiel de comprendre qu'il naît avant tout de l'échec de l'homme face à la totalité: il y a éclats de rire parce que la totalité, le sujet ne parvient qu'à la saisir de façon fragmentée, donc à la manquer, et c'est dans ce manquement même que se joue sa souveraineté paradoxale.

- L'homme souterrain ose cesser de croire au bon droit de la raison. Il clame qu'il est "parfois très agréable de briser quelqu'un». Chestov lui donne, par prosopopée, la parole: "Je ne défends pas ici la souffrance ou le bien-être, mais je suis pour mon caprice $[\ldots]$ je suis sûr que l'homme ne renoncera jamais à la vraie souffrance, c'est-à-dire à la destruction et au chaos.» Qu'est-ce que le caprice? "L'homme souterrain ne possède pas de but clair, défini. Il veut ardemment, passionnément, follement, mais il ne sait pas ce qu'il veut et ne le saura jamais.» — C'est dans son Sur Nietzsche. Volonté de chance, en 1945, que la fortune, le caprice et la chance (au simple sens de "ce qui échoit») seront le plus nettement convoqués par Bataille pour définir le comportement de l'homme sans projet(s); mais ces expériences sont déjà à l'œuvre dans les romans antérieurs.

39. Il faut cependant noter que l'impossible est aussi une notion chère au mouvement Dada, et aux surréalistes. René Char, en 1930, publie trois textes dans une revue surréaliste de Belgrade, intitulée L'impossible. Avant lui Jacques Rigaut parle de ses "désirs d'impossible» ( Jacques Rigaut», Littérature, Paris, n 17, décembre 1920), et reproche à Barrès de trouver "toutes choses possibles", tandis qu'à ses yeux, «il n'y a rien de possible, pas même le suicide» (témoignage lors de l'affaire Barrès, Littérature, Paris, n 20, août 1921. Voir Jacques Rigaut, Écrits, Paris, Gallimard, 1970, p. 21 et p. 28). Ajoutons que le premier texte paru de Rigaut s'intitule «Propos amorphes»... L'impossible, l'informe... 
— L'homme souterrain «ne pense pas; il s'agite, désespéré; il frappe de tous côtés, il cogne sa tête à tous les murs. Il s'enflamme sans cesse, il atteint les cimes les plus élevées pour se précipiter ensuite dans Dieu sait quels abîmes". C'est d'ailleurs un trait commun à tous les héros de Dostoïevski: "ses héros ne savent pas agir, ils ne savent pas créer, ils ne le veulent même pas, semble-t-il; la destruction, la mort les suivent pas à pas, afin, probablement, de ne pas donner au lecteur l'illusion même d'une conclusion». Et Chestov d'ajouter: "Tous les héros de Dostoïevski aspirent à ce qui menace de les perdre.» Lui-même "renonce à la certitude et pose comme but suprême - l'ignorance». - La destruction, le non-savoir, la perte (et la souveraineté paradoxale qui se trouve au fond de la perte): obsessions de Bataille s'il en est.

Ainsi peut-on reconnaître dans ces pages de Chestov comme la matrice non seulement de "Dirty", mais sans doute de toute l'œuvre de Bataille, fondée sur le rejet du bon sens, de la mesure, des lois, de l'idéalisme, de la raison, et sur la recherche de l'accès, dans une insatisfaction essentielle, à une autre vision, fascinée par la mort, l'indéfini, l'ignorance (le non-savoir), la perte de soi, le caprice, l'impossible - et le rire.

Chestov reviendra sur son analyse de «l'homme souterrain» dans La philosophie de la tragédie. Dostö̈evsky et Nietzsche; par là il entend ces êtres qui ont renoncé à la sensiblerie, à l'humanitarisme, à l'idéalisme, non sans une souffrance tragique. Mettant en jeu avec Dirty et Léon deux de ces êtres souterrains, donc blessés - à la main, pour Léon - et tragiques, mais se rappelant aussi que toute tragédie se fonde sur un sacrifice, Bataille, prenant Chestov au mot, raconte un sacrifice humain, dont Dirty est la victime volontaire, et place cette problématique du sacrifice, dont l'acte charnel n'est qu'une manifestation, au cœur de son œuvre romanesque.

La dissymétrie du couple, dans le récit, ne peut manquer de frapper: orgasme et audace de Dirty, passivité et impuissance de Léon. Cette dissymétrie fait songer à celle qu'évoquera Bataille dans Critique en mars 1953, dans un article intitulé «Hemingway à la lumière de Hegel ", à propos du roman The sun also rises, paru en $1926^{40}$. Si le texte d'Hemingway plut à Bataille, comme à toute une génération, c'est que, dit-il, contre la tradition de la mesure

40. Il est possible que Bataille, sur les conseils de Queneau, l'ait lu entre 1926 et 1928. En tout cas ce qu'il en dit éclaire «Dirty». Car le couple de Dirty et Léon reproduit en un sens celui de la fascinante Brett Ashley — «il n'est pas 
française: «nous aimions la fièvre et la sauvagerie, tout ce qui est immédiatement et directement», qualités qui sont aussi bien, aux yeux de Bataille, celles de Dostoïevski ${ }^{41}$. Il reste qu'avec les audaces de Dirty se manifeste la grande différence qui sépare Bataille de Dostoïevski (et encore plus nettement de Chestov): l'importance que le premier accorde à une figure féminine érotiquement scandaleuse. Faudrait-il alors oser écrire que Bataille $=$ Dostoïevski + le sexe, et avec plus... de rire? Ce qui ipso facto accomplirait aussi la soustraction du christianisme...

Pour conclure, une question naïve: les romans de Bataille ontils la puissance romanesque de ceux de Dostoïevski? Il serait présomptueux de le prétendre. Louis-René Des Forêts - qui avait lui aussi à sa manière réécrit Le sous-sol, avec Le bavard (1946) - s'y risquait pourtant, en 1958, à propos du Bleu du ciel, «où, disait-il, l'univers angoissé et nocturne des grands romans de Dostoïevski s'ouvre sur la plénitude aérienne et lumineuse du ciel de midi»; il est vrai que, dans son souci de déprécier le Nouveau Roman, il allait jusqu'à voir en Bataille "le plus grand de nos écrivains ${ }^{42}$ ", point dont on pourrait débattre, si ce genre de palmarès avait un sens. Mais sur le fond, pour satisfaire à la question posée, encore faudrait-il savoir ce qu'est le romanesque ${ }^{43}$. Si je reviens à Thibaudet, voici une réponse possible: conformément à l'origine du genre du roman — né dans "la chambre des dames" au $\mathrm{XIII}^{\mathrm{e}}$ siècle, selon lui - est romanesque un roman dans lequel «la nature féminine devient la valeur suprême ${ }^{44} »$. Or, c'est le cas chez Bataille: mais l'intérêt de son ouvre ne tient-il pas pour une large part à ce qu'il prend le mot nature à la fois dans son sens le plus impalpable (la femme comme voie d'accès à l'extase) — et dans son sens... le plus cru?

de personnage dans le livre qui ait le prestige de Brett», "en sa personne, l'ivresse est souverainement séduisante " (Hemingway à la lumière de Hegel », Critique, Paris, 1953, dans OC, t. XII, p. 246) - et de Jake Barnes, ivrogne, lui, parce qu'impuissant. Bataille fut aussi très sensible chez Hemingway à ce qu'il résume en une formule qui définit assez bien «Dirty» : la "quête de la souveraineté». En ce sens, il est logique que Dirty compare sa mère à une reine, et le prénom Léon (lion...) n’est pas immotivé.

41. Georges Bataille, «Hemingway», art. cité, p. 244.

42. Louis-René Des Forêts, La ciguë, Paris, nº 1, janvier 1958, p. 35-36.

43. Voir les réflexions de Bernard Pingaud, dans Les anneaux du manège, Paris, Gallimard, coll. «Folio/essais», 1992, et La bonne aventure, Paris, Seuil, 2007; ainsi que Gilles Declercq et Michel Murat (sous la dir. de), Le romanesque, Paris, Presses de la Sorbonne nouvelle, 2004.

44. Albert Thibaudet, Réflexions, ouvr. cité, p. 110. 